\title{
Fall Prevention Education Reduces the Falling Rate on the Osteoporosis Patients Treated with Zoledronic Acid
}

\author{
Ermei Jie', Jinmei Deng2* \\ ${ }^{1}$ Department of Endocrinology, The First Affiliated Hospital, Sun Yat-sen University, Guangzhou, China \\ ${ }^{2}$ Outpatient Clinic of Internal Medicine Department, The First Affiliated Hospital, Sun Yat-sen University, Guangzhou, China \\ Email: *309346496@qq.com, 13710551015@139.com
}

How to cite this paper: Jie, E.M. and Deng, J.M. (2019) Fall Prevention Education Reduces the Falling Rate on the Osteoporosis Patients Treated with Zoledronic Acid. Case Reports in Clinical Medicine, 8, 222-230.

https://doi.org/10.4236/crcm.2019.88027

Received: June 10, 2019

Accepted: August 12, 2019

Published: August 15, 2019

Copyright $\odot 2019$ by author(s) and Scientific Research Publishing Inc. This work is licensed under the Creative Commons Attribution International License (CC BY 4.0).

http://creativecommons.org/licenses/by/4.0/

\section{Abstract}

Objective: Falls are one of the most common direct causes of fractures which are major causes of morbidity and mortality in osteoporosis patient. There are many factors related to falls, by interfering of fall prevention education on the patients with osteoporosis, our study is to investigate whether fall prevention education can reduce the falling rate on the osteoporosis patients treated with zoledronic acid. Methods: A total of 178 eligible female patients who first visited our osteoporosis department during January 2016 to June 2017 were invited to participate in this study, and all participants were randomly divided into an observation group (92 cases) and an intervention group (86 cases). All patients were administrated zoledronic acid once and received a questionnaire survey about factors related to falls at the beginning and a year later. The patients in the observation group only received regular orders of adopting a healthy lifestyle while those in the intervention group received fall prevention assessment and education, and a telephone follow-up and reinforced fall prevention education a month after discharge. A year later, relevant data regarding the falls of each patient of both groups during the year and the data of the questionnaire survey were collected for intergroup comparison. Results: The difference of the improvement of fall risk factors between the two groups is statistically significant $(\mathrm{P}<0.05)$, except the factor of diabetes without treatment. In the intervention group, $5.8 \%$ fell at least once, and $19.6 \%$ of the patients in the observation group reported the same during the year. Comparing the cases of falls of the two groups, the difference shows statistical significance $(P=0.006)$. Conclusion: For an osteoporosis patient treated with zoledronic acid, fall prevention education is an effective method to reduce the risk of falling, which would result in a lower risk of fractures 
and a better prognosis.

\section{Keywords}

Fall, Prevention, Education, Osteoporosis, Zoledronic Acid

\section{Introduction}

Osteoporosis is a metabolic bone disease featuring low bone mass and microstructural deterioration of bone tissue, leading to an increased risk of fragile fractures that have high rates of fatality and disability and severely affect a patient's quality of life [1]. Falls are one of the most common direct causes of fractures. It is reported that in China, $21 \%-23 \%$ males and $43 \%-44 \%$ females of the community-dwelling population aged 65 and above fall at least once [2]. An osteoporosis patient is exposed to the risk of falling associated with such internal factors as age, and balance problems and underlying diseases caused by osteoporosis, as well as many external factors, like slippery footwear, a dangerous living environment, and intense exercise [3]. Multifaceted podiatric interventions, which include appropriate footwear and importantly patient education, may have the capacity to reduce falls in older adults [4]. Although the elderly perceived that fall could be avoided, they did not know how to prevent it. The intervention that focuses on environmental modification, balancing enchantment, and education on fall prevention is highly required [5]. This justifies the need to provide osteoporosis patients with fall prevention education along with medication. Clinical attention is paid to the treatment of anti-osteoporosis drugs, while the education of preventing falls is often ignored. This study aims at reducing the risk of falling posed to osteoporosis patients based on effective fall prevention education along with zoledronic acid treatment.

\section{Materials and Methods}

\subsection{Clinical Materials}

Clinical data of 178 female patients who first visited our osteoporosis clinic from January 2016 to June 2017 were collected. These patients were between the age of 50 to 80 , with the mean age of $68.1 \pm 9.0$. Dual-energy X-ray absorptiometry (DEXA) was used to test each patient's bone mineral density (BMD) of the lumbar spine and the femur before making an initial diagnosis and the test results were considered diagnosis and inclusion criteria. Inclusion criteria: A patient was eligible for inclusion if 1 ) her bone mineral density $\mathrm{T}$-score was no greater than -2.5 or the $\mathrm{T}$-score was less than -1.0 with preexisting fragility fractures and definite systemic symptoms such as back pain or height loss; 2) she had never been administrated zoledronic acid before; 3 ) she was a clear-minded and right-thinking individual who agreed to participate in this study and signed the informed consent; 4) she demonstrated proven learning ability. 


\subsection{Methods}

\subsubsection{Overview}

A total of 178 Eligible participants were randomly divided into an observation group (92 cases) and an intervention group (86 cases). All patients were administrated zoledronic acid once and received a questionnaire survey about factors related to falls at the beginning and a year later. The patients in the observation group only received regular orders of adopting a healthy lifestyle while those in the intervention group received fall prevention assessment and education. Combining with the results of the questionnaire survey, there is rectification guidance to the patients. Then there was a telephone follow-up and reinforced fall prevention education a month after discharge. A year later, relevant data regarding the falls of each patient of both groups during the year and the data of the questionnaire survey were collected for intergroup comparison.

\subsubsection{Intervention Plan}

1) Assessing Potential Risk Factors with the questionnaire for patients with osteoporosis which is shown at the end of the article.

2) Fall prevention education on the following risk factors to the intervention group, the contents of education are as follows.

Physiological factors: damaged or degenerative gait and functions of the balanced system, sensing system, central nervous system, or skeletal musculature.

Underlying diseases: neurological, cardiovascular, or eye diseases that may affect an individual's balanced system, stability, and physical coordination.

Medication and side effects: medication, dosage, and compound prescriptions possibly associated with a patient's fall(s), such as psychotropic drugs, cardiovascular drugs, hypoglycemic drugs, non-steroidal anti-inflammatory drugs (NSAIDs), antiparkinsonian agents, and dopamine drugs.

Psychological factors: dejection, depression of spirits, anxiety, and other mentalities that may increase the risk of falls and injury. A fear of falling will affect one's capacity, gait, and balance system, thereby increasing the risk of getting injured by a fall.

Environmental risk factors: dim light in a house, slippery/bumpy floor, a bed or another furniture at an inconvenient height, an inappropriate layout of furniture, no handrail in a bathroom, unfitting/slippery footwear, a lack of necessary walking aids, and rough roads in places that an individual visits a lot.

Social factors: a patient's educational background, living status (living alone or not), social communication ability, and social network.

3) Combining with the results of the survey, there is rectification guidance to the patients of intervention group as follows:

a) If your basis disease has not given treatment, you should go to see a specialist, and take the prescribed treatment.

b) If you are inconvenient to walk, you should use the auxiliary device.

c) If you can't see things clearly, you should wear glasses.

d) If your shoes are not appropriate and antiskid, you should wear appropriate 
and antiskid shoes.

e) If the light is not bright enough at your home, you should replace the appropriate light tube.

f) If the floor of your house is not anti-skidding, you should use slip-resistant mats in and outside of bathroom to prevent slips and falls.

g) If there is no anti-skidding armrest in the toilet of your house, you should install the slippery armrest.

h) If your home bed is too high, and you are easy to fall down, you should adjust the height of your bed.

i) If the path that you often go out is not smooth, you should choose another safe route.

\subsection{Effectiveness Evaluation}

Telephone follow-ups were made to investigate the overall incidence of falls of each group in a year, and a comparative analysis was performed on this basis. The results of the questionnaire survey were used to compare the improvement of fall factors between the two groups after one year.

\section{Statistical Analysis}

The t-test was employed in the intergroup comparison of the patients' general information and their questionnaire scores before treatment while the chi-square test was used for the intergroup comparison of the enumeration data. $\mathrm{P}<0.05$ indicates a difference of statistical significance.

\section{Results}

According to Table 1 and Table 2, there is no statistically significant difference between the two groups in age, bone mineral density, and underlying diseases and other fall factors according to the questionnaire for patients with osteoporosis $(\mathrm{P}>0.05)$, which indicates a high degree of comparability.

As shown in Table 3, the improvement of fall risk factors of the observation group was relatively small; in contrast, the improvement of fall risk factors of the intervention group was obvious. The difference of the improvement of fall risk factors between the two groups is statistically significant $(P<0.05)$, except the factor of diabetes without treatment. In other words, fall prevention education can markedly improve the factors related to falls.

Table 1. Patients' general information.

\begin{tabular}{cccc}
\hline & Observation Group & Intervention Group & P-Value \\
\hline Number of Patients & 92 & 86 & \\
Age & $68.5 \pm 8.7$ & $69.2 \pm 9.2$ & 0.564 \\
BMD $_{\text {L2-4 }}$ & $0.787 \pm 0.119$ & $0.797 \pm 0.110$ & 0.555 \\
BMD $_{\text {hip }}$ & $0.603 \pm 0.144$ & $0.593 \pm 0.092$ & 0.580 \\
\hline
\end{tabular}

Note: The age unit is year; BMD unit is $\mathrm{mg} / \mathrm{cm}^{2}$. 
Table 2. Comparison of fall factors between the two groups.

\begin{tabular}{ccccc}
\hline & $\begin{array}{c}\text { Observation Group } \\
(\mathrm{n} / \%)\end{array}$ & $\begin{array}{c}\text { Intervention Group } \\
(\mathrm{n} / \%)\end{array}$ & $\mathrm{X}^{2}$ & P-Value \\
\hline $\begin{array}{c}\text { Nervous system diseases } \\
\text { Nervous system diseases } \\
\text { without treatment }\end{array}$ & $18(19.6 \%)$ & $15(17.4 \%)$ & 0.133 & 0.716 \\
$\begin{array}{c}\text { Cardiovascular diseases } \\
\text { Cardiovascular diseases }\end{array}$ & $8(8.7 \%)$ & $9(10.5 \%)$ & 0.161 & 0.688 \\
without treatment & $12(13 \%)$ & $11(12.8 \%)$ & 0.003 & 0.96 \\
$\quad$ Diabetes & $25(24.5 \%)$ & $26(30.2 \%)$ & 0.773 & 0.379 \\
$\begin{array}{c}\text { Diabetes without treatment } \\
\text { Inconvenient to walk and } \\
\text { no auxiliary device }\end{array}$ & $5(5.4 \%)$ & $4(4.7 \%)$ & 0.057 & 0.812 \\
Visual impairment but & $10(10.9 \%)$ & $8(9.3 \%)$ & 0.120 & 0.729 \\
no glasses & $32(34.8 \%)$ & $27(31.4 \%)$ & 0.230 & 0.631 \\
Unsuitable shoes & $6(6.5 \%)$ & $7(8.1 \%)$ & 0.172 & 0.678 \\
$\quad$ Unbright light & $5(5.4 \%)$ & $3(3.5 \%)$ & 0.392 & 0.531 \\
No anti-skidding floor & $8(8.7 \%)$ & $10(11.6 \%)$ & 0.420 & 0.517 \\
No anti-skidding armrest & $30(32.6 \%)$ & $27(31.4 \%)$ & 0.030 & 0.862 \\
Home bed too high & $4(4.3 \%)$ & $6(7.0 \%)$ & 0.579 & 0.447 \\
$\begin{array}{c}\text { Unsmooth and unsafe rout } \\
\text { Uns }\end{array}$ & $6(6.5 \%)$ & $7(8.1 \%)$ & 0.172 & 0.678 \\
\hline
\end{tabular}

Table 3. Comparison of improvement of fall risk factors between the two groups after one year.

\begin{tabular}{|c|c|c|c|c|}
\hline & $\begin{array}{c}\text { Observation Group } \\
(\mathrm{n} / \%)\end{array}$ & $\begin{array}{c}\text { Intervention Group } \\
(\mathrm{n} / \%)\end{array}$ & $\mathrm{X}^{2}$ & P-Value \\
\hline $\begin{array}{l}\text { Nervous system diseases } \\
\text { without treatment }\end{array}$ & $2(25 \%)$ & $7(77.8 \%)$ & 4.735 & 0.03 \\
\hline $\begin{array}{c}\text { Cardiovascular diseases } \\
\text { without treatment }\end{array}$ & $2(16.7 \%)$ & $9(81.8 \%)$ & 9.763 & 0.002 \\
\hline Diabetes without treatment & $2(40 \%)$ & $4(100 \%)$ & 3.6 & 0.058 \\
\hline $\begin{array}{l}\text { Inconvenient to walk } \\
\text { and no auxiliary device }\end{array}$ & $3(30 \%)$ & $7(87.5 \%)$ & 5.95 & 0.015 \\
\hline $\begin{array}{l}\text { Visual impairment } \\
\text { but no glasses }\end{array}$ & $4(12.5 \%)$ & $23(85.2 \%)$ & 31.171 & 0.000 \\
\hline Unsuitable shoes & $1(16.7 \%)$ & $6(85.7 \%)$ & 6.198 & 0.013 \\
\hline Unbright light & $1(20 \%)$ & $3(100 \%)$ & 4.8 & 0.028 \\
\hline No anti-skidding floor & $1(12.5 \%)$ & $8(80 \%)$ & 8.1 & 0.004 \\
\hline No anti-skidding armrest & $2(6.7 \%)$ & $17(63 \%)$ & 20.267 & 0.000 \\
\hline Home bed too high & $0(0 \%)$ & $5(83.3 \%)$ & 6.667 & 0.01 \\
\hline Unsmooth and unsafe rout & $0(0 \%)$ & $4(57.1 \%)$ & 4.952 & 0.026 \\
\hline
\end{tabular}

As shown in Table 4, the observation group has an incidence of falls of $19.6 \%$ during the year; in contrast, the incidence of falls of the intervention group is $5.8 \%$, considerably lower than that of the observation group. The difference between the two groups is statistically significant $(\mathrm{P}<0.05)$. In other words, fall 
Table 4. Comparison of the two groups' incidence rates of fall events in a year.

\begin{tabular}{cccc}
\hline & Non-Falling Cases & Falling Cases & Incidence of Falls \\
\hline Observation Group & 74 & 18 & $19.6 \%$ \\
Intervention Group & 81 & 5 & $5.8 \%$ \\
\hline
\end{tabular}

Note: The incidence rates of falls in the two groups are examined by the chi-squared test and the result is $\mathrm{X}^{2}=$ 7.47, and $\mathrm{P}=0.006$.

prevention education can markedly reduce the incidence of falls in elderly patients with osteoporosis who are treated with zoledronic acid.

\section{Discussion}

As population aging advances, age-related osteoporosis has become a common disease. Against this backdrop, treatment for osteoporosis that increases bone mineral density and reduces the risk of fractures has been heatedly discussed by the mass [6]. Falls act as an independent risk factor of osteoporotic fractures, and fall prevention is considered as an effective method to remarkably lower the incidence of fractures [7]. There are foreign studies reporting that $30 \%-40 \%$ of the community-dwelling population aged 65 and above in their countries fall at least once every year, and nearly $50 \%$ of these individuals fall more than once. In China, up to $41 \%$ of the elderly fall more than once a year [8]. Falls in the elderly are a major health problem due to their traumatic and psychosocial complications which may lead to a loss of autonomy and a state of dependency [9]. Accidental falls are a leading cause of injury and death in older adults. Most hip fracture survivors do not regain their former levels of activity or mobility and so are at increased risk of further falls [10]. Osteoporotic proximal femoral fractures associated to falls are a major health burden in the ageing society. In the UK, fragility hip fractures cost NHS approximately $£ 1.1$ billion [11]. So falls prevent eduction is very important for the osteoporosis patients, which is in order to reduce the incidence of falls and thus reduce the risk of fracture.

Since there are a great variety of risk factors related to fall events, including both internal and external ones, a fall prevention program should offer elaborate and feasible education to minimize the risk of falls and injury. Considering that an osteoporosis clinic mainly provides services for elderly patients having slow reflexes and varied underlying diseases, detailed professional fall prevention education is required in addition to the order of developing a healthy lifestyle during a consultation. Also, careful rectifications are recommended to protect the patients from relevant risk factors; to this end, a specific rectification plan should be formulated for effective implementation. In this study, factors related to fall which were easy to be improved and also were very important were selected for intervention. The result shows that proper intervention to prevent fall events through education can promote rectifications and reduce the incidence of falls. Comparing to the observation group, the intervention group has a significantly lower incidence of falls, which indicates 
favorable outcomes of fall prevention education. Yet, it should be noted that a lower incidence rate was reported by the observation group compared to other studies. This is probably because the anti-osteoporosis drug helps increase bone mineral density and improves the balance system. Zoledronic acid is a third-generation bisphosphonates medication. In Lin et al., it is demonstrated that zoledronic acid, as a once-yearly infusion, has notably increased the bone mineral density (lumbar spine $+5.8 \%$; femoral neck $+2.9 \%$; Ward's triangle $+5.2 \%$; trochiter $+5.3 \%$; total hip $+3.9 \%)$ and reduced the risk of fractures in postmenopausal osteoporosis cases [12].

As prevention is better than cure, health education makes an essential part of clinical nursing. A pertinent, goal-oriented program can help patients broaden their knowledge of healthcare and encourage a shift towards healthy behaviors and a change in lifestyle for their own good, thereby preventing diseases, promoting recovery, highlighting the humanistic spirit, and improving doctor-patient communication [13]. Fall prevention education is an osteoporosis patient-dedicated program that effectively reduces the incidence rates of falls and fractures. Combining anti-osteoporosis drugs, it helps improve patient compliance and prognosis [14].

\section{Limitations}

The use of a randomized control group may bring a bias to the statistical results because the underlying diseases are less controllable. The answers to the questionnaires used in the study depend on the subjective factors of the patients may be deviations from the results of the study. There are so many factors related to fall that can't all be involved, which may influence the results of the study.

\section{Conclusion}

Falls are one of the most common direct causes of fractures which are major causes of morbidity and mortality in osteoporosis patient. Reducing the incidence of falls can reduce the incidence of fracture in patients with osteoporosis. There are many factors related to falls, in this study, factors which were easy to be improved were selected for intervention. The result shows that fall prevention education significantly improves the factors related to falls and lowers the incidence of fall events occurring to elderly patients with osteoporosis. A treatment plan including a comprehensive health education program is cost saving and efficacious, thus worth promotion and publicity among osteoporosis clinics. The study reminds medical and nursing staff to pay attention to the education of preventing falling.

\section{Conflicts of Interest}

The authors declare no conflicts of interest regarding the publication of this paper. 


\section{References}

[1] Chinese Society of Osteoporosis and Bone Mineral Research, Chinese Medical Association (2017) A Guide to Diagnosis and Treatment of Primary Osteoporosis (2017 Edition). Chinese Journal of Endocrinology and Metabolism, 33, 890-913.

[2] Zhang, W., Cao, B.Y. and Gong, C.H.X. (2008) Study on the Risk Factor of Osteoporosis in Beijing. Chinese Journal of Osteoporosis, 14, 519-520, 514.

[3] Yin, Y. and Ren, J.G. (2017) The Influence Factors of Older Adults' Falls in Urban China. Lanzhou Journal, 17, 157-173.

[4] Hatton, A.L. and Rome, K. (2019) Falls, Footwear, and Podiatric Interventions in Older Adults. Clinics in Geriatric Medicine, 35, 161-171.

https://doi.org/10.1016/j.cger.2018.12.001

[5] Maneeprom, N., Taneepanichskul, S. and Panza, A. (2018) Falls among Physically Active Elderly in Senior Housings, Bangkok, Thailand: Situations and Perceptions. Clinical Interventions in Aging, 13, 2149-2159. https://doi.org/10.2147/CIA.S175896

[6] Yin, X.X., Song, C.L. and Du, G.H. (2015) The Effect of Anti-Osteoporosis Therapy on Bone Mineral Density and Fracture Rate in Patients with Primary Osteoporosis. Chinese Journal of Osteoporosis, 15, 421-423.

[7] Zhou, J., et al. (2017) Prevalence of Osteoporosis, Osteopenia, Fall and Brittle Fracture and Their Associated Factors in Men Aged 80 Years and Over. Chinese Journal of Geriatrics, 36, 868-871.

[8] Yu, P.L., et al. (2006) Incidence of Falls in a Community-Dwelling Older Population in Beijing. Chinese Journal of Geriatrics, 25, 305-308.

[9] Kechaou, I., Cherif, E., Sana, B.S., Boukhris, I. and Hassine, L.B. (2019) Traumatic and Psychosocial Complications of Falls in the Elderly in Tunisia. Pan African Medical Journal, 32, 92. https://doi.org/10.11604/pamj.2019.32.92.16667

[10] Handoll, H.H., Sherrington, C. and Mak, J.C. (2011) Interventions for Improving Mobility after Hip Fracture Surgery in Adults. Cochrane Database of Systematic Reviews, No. 3, CD001704. https://doi.org/10.1002/14651858.CD001704.pub4

[11] Leal, J., Gray, A.M., Prieto-Alhambra, D., Arden, N.K., Cooper, C., Javaid, M.K. and Judge, A. (2016) Impact of Hip Fracture on Hospital Care Costs: A Population-Based Study. Osteoporosis International, 27, 549-558.

https://doi.org/10.1007/s00198-015-3277-9

[12] Lin, H., et al. (2011) Effect of 5 mg Zoledronic Acid on Bone Mineral Density in Women with Postmenopausal Osteoporosis. Chinese Journal of Orthopaedics, 31, 1331-1336.

[13] Peng, L.J. and Xu, X.X. (2018) Application of Individualized Health Education in Diagnosis and Treatment of Fracture. Chinese Journal of Pharmacy Medicine, 25, 1070-1072.

[14] Li, W.Y., Liu, Y.T. and Xu, Y. (2016) The Effect of Continuity of Care on Medication Compliance and Quality of Life of Elderly Osteoporosis Patients after Discharge. Journal of Military Surgeon in Southwest China, 18, 583-585. 


\section{Questionnaire for Patients with Osteoporosis}

Name: gender: age:

Contact number: group:

1) Do you have any underlying diseases as follow?

A) Nervous system diseases, such as stroke, Parkinson's dizziness.

B) Cardiovascular diseases, such as hypertension, heart failure.

C) Diabetes.

2) If there are any of the diseases above, is it given specialist treatment?
A) Yes.
B) No.

3) Are you convenient to walk? Do you need to use the auxiliary device?

A) Yes, I am convenient to walk, do not need to use the auxiliary device.

B) No, I am inconvenient to walk, and have used the auxiliary device.

C) No, I am inconvenient to walk, but don't want to use the auxiliary device.

4) Do you see things clearly? Do you need to wear glasses?

A) Yes, I can see things clearly, and do not need to wear glasses.

B) No, I can't see things clearly and have worn glasses.

C) No, I can't see things clearly, but I don't want to wear glasses.

5) Do you wear appropriate and antiskid shoes?
A) Yes.
B) No.

6) Is the light bright enough at you home?
A) Yes.
B) No.

7) Is the floor of your house anti-skidding?
A) Yes.
B) No.

8) Is there any anti-skidding armrest in the toilet of your house?
A) Yes.
B) No.

9) Is your home bed too high, and you are easy to fall down?
A) Yes.
B) No.

10) Is the path that you often go out smooth? Do you often fall down while you go out through the path?
A) Yes, it is smooth.
B) No, it is unsmooth, and I often fall down while I go out through the path. 